\title{
Clinical significance of nuclear factor erythroid 2-related factor 2 in patients with chronic obstruc- tive pulmonary disease
}

\author{
Woo Ho Ban ${ }^{1,}$, Hyeon Hui Kang, ${ }^{1,}$, In Kyoung Kimㄹ, Jick Hwan $\mathrm{Ha}^{1}$, Hyonsoo Joo ${ }^{1}$, Jong Min Lee ${ }^{1}$, \\ Jeong Uk Lim ${ }^{1}$, Sang Haak Lee ${ }^{1}$, and Chin Kook Rhee ${ }^{2}$
}

\begin{abstract}
${ }^{1}$ Division of Pulmonary, Critical Care and Sleep Medicine, Department of Internal Medicine, College of Medicine, St. Paul's Hospital, The Catholic University of Korea, Seoul; ${ }^{2}$ Division of Pulmonary, Allergy and Critical Care Medicine, Department of Internal Medicine, College of Medicine, Seoul St. Mary's Hospital, The Catholic University of Korea, Seoul, Korea
\end{abstract}

Received: January 10, 2017

Revised : August 6, 2017

Accepted: August 31, 2017

\section{Correspondence to}

\section{Chin Kook Rhee, M.D.}

Division of Pulmonary, Aller-

gy and Critical Care Medicine,

Department of Internal Medicine,

College of Medicine, Seoul St.

Mary's Hospital, The Catholic

University of Korea, 222

Banpo-daero, Seocho-gu, Seoul

06591, Korea

Tel: $+82-2-2258-6067$

Fax: $+82-2-599-3589$

E-mail: chinkook@catholic.ac.kr

*These authors contributed equally to this work.
Background/Aims: Several studies have identified a role for nuclear factor erythroid 2-related factor 2 ( $\mathrm{Nrf} 2$ ) in the development of chronic obstructive pulmonary disease (COPD). However, the relationship between the plasma Nrf2 level and the extent of systemic inflammation associated with COPD status remains unclear. Methods: Patients diagnosed with COPD were recruited from St. Paul's Hospital, The Catholic University of Korea, between July 2009 and May 2012. Patients were classified into two groups according to the severity of their symptoms on initial presentation, a COPD-stable group $(\mathrm{n}=25)$ and a COPD-exacerbation group $(\mathrm{n}=$ 30). Seventeen patients were enrolled as a control group $(n=17)$. The plasma levels of Nrf2 and other systemic inflammatory biomarkers, including interleukin 6 (IL-6), surfactant protein D (SP-D), and C-reactive protein (CRP), were measured. We collected clinical data including pulmonary function test results, and analyzed the relationships between the biomarker levels and the clinical parameters. Results: Plasma Nrf2 and CRP levels significantly increased in a stepwise manner with an increase in inflammatory status (control vs. COPD-stable vs. COPD-exacerbation) $(p=0.002, p<0.001)$. Other biomarkers of systemic inflammation (IL-6, SP-D) exhibited similar tendencies, but significant differences were not apparent. Furthermore, we observed negative correlations between the plasma level of Nrf2 and both the forced expiratory volume in 1 second $\left(\mathrm{FEV}_{1}\right)(r=-0.339, p=0.015)$ and the forced expiratory ratio $\left(\mathrm{FEV}_{1} /\right.$ forced vital capacity $\left.[\mathrm{FVC}]\right)(r=-0.342, p=0.014)$. However, CRP level was not correlated with any measured parameter.

Conclusions: Plasma Nrf2 levels gradually increased in line with disease severity and the extent of systemic inflammation in patients with COPD.

Keywords: Lung diseases; Chronic obstructive; NF-E2-related factor 2; Biomarkers

\section{INTRODUCTION}

Chronic obstructive pulmonary disease (COPD) is a persistent progressive disease of the airways that features a decline in lung function and symptoms of dyspnea. COPD is associated with tremendous economic burden and is predicted to become the fourth-leading cause of death worldwide by the year 2030 [1]. In 2010, the projected annual cost of COPD in the United States, both direct and indirect, was $\$ 49.9$ billion [2]. As COPD is not a single pulmonary disease but rather a systemic disease that accompanies other comorbidities, including cardiovascular disease and diabetes, COPD is associated with heavy use of resources and high-level expenditures [3]. 
Continuous exposure to oxidative stress arising from smoking and air pollution causes chronic inflammation of the airways and whole body and is widely accepted as the main pathophysiology of COPD. Therefore, scientific approaches to COPD have moved from consideration of a single pulmonary disease to a systemic disease.

The human body has several anti-oxidative systems that respond to oxidative stress, and many enzymes and molecules engage in these systems. Among them, nuclear factor erythroid 2-related factor 2 (Nrf2) plays a major protective role in the lungs and whole body against oxidative stress. Nrf2 is a transcription factor that scavenges molecules exerting oxidative stress through the activation of antioxidant response elements (AREs); thus, regulating antioxidant genes [4]. Several studies have identified a relationship between the expression of $\mathrm{Nrf}_{2}$ and the development of COPD. Hence, Nrf2 might be an important molecule for understanding the various aspects of COPD and even for disease prevention [5].

Exacerbation of COPD has been defined as a "sustained worsening of the patient's condition, from a stable state and beyond normal day-to-day variations" [6]. Many studies have revealed that COPD-exacerbation is closely related to excessive inflammation in response to increased oxidative stress [7]. It is thus important to understand the mechanism of exacerbation; this will help physicians understand the inflammatory process more clearly and prevent COPD progression. Thus, many researchers have sought to identify reliable biomarkers predicting and detecting exacerbation. However, to date, no definitive biomarkers have been identified.

In this study, we assessed oxidative stress and systemic inflammation expressed as the plasma level of Nrf2 and other systemic inflammatory markers, including interleukin 6 (IL-6), surfactant protein D (SP-D), and C-reactive protein (CRP), in both stable and exacerbation states of COPD patients, and compared them with those in controls. Additionally, we estimated the value of $\mathrm{Nrf2}$ as a potential biomarker for disease monitoring, through the analysis of correlations between the plasma Nrf2 level and other clinical parameters associated with COPD.

\section{METHODS}

\section{Study population and data collection}

Patients diagnosed with COPD by the Global Initiative for Chronic Obstructive Lung Disease (GOLD) criteria [8] were recruited into this study from St. Paul's Hospital, The Catholic University of Korea, between July 2009 and May 2012. Among these, 25 patients had stable COPD and 30 had exacerbation of COPD. Exacerbation was defined "a worsening of respiratory symptoms, requiring treatment with oral corticosteroids or antibiotics, or admission" [9]. Some of the patients who visited the outpatient clinic of St. Paul's Hospital due to minor respiratory symptoms such as common cold and cough agreed to participate in this research and were assigned to the control group. We excluded patients who had other significant pulmonary diseases such as pneumonia, lung cancer, and tuberculosis. After obtaining written informed consent, we collected blood samples at the outpatient clinic or in the emergency room before administration of oral corticosteroids and/or antibiotics, and before any other treatment. We retrospectively reviewed each patient's clinical data and gathered information on age, sex, body mass index, past medical history, and smoking history. Pulmonary function tests were performed according to the American Thoracic Society/ European Respiratory Society standardization guidelines [10]. Data on pulmonary function parameters, such as forced vital capacity (FVC), forced expiratory volume in 1 second $\left(\mathrm{FEV}_{1}\right)$, forced expiratory ratio $\left(\mathrm{FEV}_{1} / \mathrm{FVC}\right)$, and the diffusion capacity of the lung for carbon monoxide (DLCO), were collected. This study was approved by the Institutional Review Board Ethics Committee of St. Paul's Hospital, The Catholic University of Korea (PC14SISIoo58).

\section{Measurement of biomarkers}

Blood samples were collected from all study patients. Blood was centrifuged at 3,000 rpm for 15 minutes at $4^{\circ} \mathrm{C}$. Plasma samples were immediately stored at $-80^{\circ} \mathrm{C}$ until required for analysis. Nrf2 levels $(\mathrm{ng} / \mathrm{mL})$ were assayed using a commercially available DNA-binding enzyme-linked immunosorbent assay (ELISA) (TransAM Nrf-2, Active Motif, Carlsbad, CA, USA). The concentration of IL-6 (pg/mL) was determined by a Human IL-6 Quantikine ELISA kit (R\&D Systems, Minneapolis, MN, 
USA), in accordance with the manufacturer's instructions. SP-D levels $(\mathrm{ng} / \mathrm{mL})$ were also measured with an ELISA kit (BioVendor Inc., Brno, Czech Republic). All assays were measured in plasma and reactions were read using an ELISA plate reader at $450 \mathrm{~nm}$. Serum CRP levels were measured using a commercially available kit (Nanopia CRP, Sekisui Medical Co. Ltd., Tokyo, Japan), in accordance with the manufacturer's directions.

\section{Statistical analysis}

Statistical analyses were performed using the IBM SPSS Statistics version 20.0 for Windows (IBM Co., Armonk, NY, USA). The chi-square test was used to compare the proportions of categorical variables. A comparison of continuous variables for each group (control vs. COPD-stable vs. COPD-exacerbation) was performed via one-way analysis of variance or Kruskal-Wallis test with Bonferroni's method for post hoc analysis. The Jonckheere-Terpstra test was used to assess trends in plasma biomarker levels by disease status. The Mann-Whitney test was used to compare differences in continuous variables between COPD groups in a non-parametric manner. Pearson's correlation coefficient was used to assess the relationships between biomarkers and other clinical parameters of the population. The two-sided significance level was set at a $p<0.05$.

\section{RESULTS}

\section{Clinical characteristics of the study population}

A total of 55 patients with COPD were recruited into this study and were classified into two groups according to the severity of their symptoms on initial presentation: the COPD-stable group $(\mathrm{n}=25)$ and COPD-exacerbation group $(\mathrm{n}=30)$. Seventeen patients were enrolled as the control group $(n=17)$. Table 1 shows the clinical characteristics of each group in detail.

The mean patient age was older in the COPD groups than in the control group. There was no significant difference in body composition among the three groups. Patients with a history of tuberculosis were more abundant in both COPD groups than in the control group. Three cases of osteoporosis were reported in the COPD-exacerbation group. No significant differences were observed among the three groups in terms of smoking history. The number of exacerbations in the past year was significantly higher in the COPD-exacerbation group than in the COPD-stable group.

\section{Comparison of pulmonary function parameters among the COPD groups and the control group} The pulmonary function parameters of the COPD groups and the control group are presented in Table 1. Compared with the control group, the COPD groups tended to have worse respiratory function. There were significant differences in $\mathrm{FEV}_{1}(\%)$ and $\mathrm{FEV}_{1} / \mathrm{FVC}(\%)$ among the three groups. No significant differences were detected in the FVC (\%) and DLCO (\%) values.

\section{Systemic inflammatory and oxidative stress bio- marker levels in the COPD groups and the control group}

The Nrf2, IL-6, SP-D, and CRP levels of the three groups are shown in Table 2 and Fig. 1. All of the systemic inflammatory marker levels were highest in the COPD-exacerbation group, followed by the COPD-stable group and, finally, the control group. Significant differences were detected in only the Nrf2 and CRP levels and notable distinctions of both parameters were observed between the control group and the COPD-exacerbation group (Fig. 1A and 1D). However, significant stepwise increases in line with the inflammatory status (control vs. COPD-stable vs. COPD-exacerbation) were noted in both the Nrf2 and CRP levels.

\section{Correlations between $\mathrm{Nrf2}$ and other clinical param- eters in COPD}

Fig. 2 shows the correlation of plasma Nrf2 levels with other clinical parameters in COPD. A significant negative correlation was found between $\mathrm{FEV}_{1}$ (\%) and the plasma Nrf2 level (Fig. 2A). Additionally, there was a significant negative correlation between $\mathrm{FEV}_{1} / \mathrm{FVC}(\%)$ and the Nrf2 level (Fig. 2B), whereas no significant correlation was found between the CRP and Nrf2 levels (Fig. 2C). The serum CRP level did not show any correlation with either $\mathrm{FEV}_{1}$ (\%) or $\mathrm{FEV}_{1} / \mathrm{FVC}(\%)$ (data not shown).

\section{DISCUSSION}

In this study, we investigated the expression of plasma 
Table 1. Patient characteristics and pulmonary function parameters

\begin{tabular}{|c|c|c|c|c|}
\hline Variable & $\begin{array}{l}\text { Control } \\
(\mathrm{n}=17)\end{array}$ & $\begin{array}{l}\text { COPD-stable } \\
\qquad(\mathrm{n}=25)\end{array}$ & $\begin{array}{l}\text { COPD-exacerbation } \\
(\mathrm{n}=30)\end{array}$ & $p$ value \\
\hline Age & $61.06 \pm 14.36$ & $71.88 \pm 11.27$ & $73.43 \pm 8.17$ & 0.001 \\
\hline Male sex & $15(88.2)$ & $18(72.0)$ & $27(90.0)$ & 0.216 \\
\hline BMI, kg/m² & $22.82 \pm 3.17$ & $21.52 \pm 2.95$ & $21.83 \pm 4.22$ & 0.398 \\
\hline \multicolumn{5}{|l|}{ Comorbidities } \\
\hline Diabetes mellitus & $9(52.9)$ & $8(32.0)$ & $9(30.0)$ & 0.270 \\
\hline Hypertension & $5(29.4)$ & $14(56.0)$ & $10(33 \cdot 3)$ & 0.148 \\
\hline History of tuberculosis & $1(5 \cdot 9)$ & $9(36.0)$ & $14(46.7)$ & 0.016 \\
\hline $\begin{array}{l}\text { History of cerebrovascular } \\
\text { disease }\end{array}$ & o & $3(12.0)$ & $1(3 \cdot 3)$ & 0.280 \\
\hline History of myocardial infarction & $1(5 \cdot 9)$ & $3(12.0)$ & $5(16.7)$ & 0.539 \\
\hline Osteoporosis & 0 & 0 & $3(10.0)$ & 0.175 \\
\hline $\begin{array}{l}\text { Smoking history, never/former/ } \\
\text { current smoker }\end{array}$ & $5(29.4) / 7(41.2) / 5(29.4)$ & $4(16.0) / 12(48.0) / 9(36.0)$ & $3(10.0) / 21(70.0) / 6(20.0)$ & 0.231 \\
\hline Smoking pack-years & $31.17 \pm 26.61$ & $30.58 \pm 21.95$ & $35 \cdot 52 \pm 28.02$ & 0.791 \\
\hline $\begin{array}{l}\text { No. of exacerbations in the past } \\
\text { year }\end{array}$ & & $1.04 \pm 1.31$ & $1.60 \pm 1.07$ & 0.014 \\
\hline \multicolumn{5}{|l|}{ Pulmonary function parameters } \\
\hline FVC, \% predicted & $98.40 \pm 21.62$ & $86.13 \pm 28.04$ & $72.46 \pm 22.98$ & 0.057 \\
\hline $\mathrm{FEV}_{1}, \%$ predicted & $112.80 \pm 27.59$ & $69.54 \pm 27.39$ & $45.25 \pm 21.57$ & $<0.001$ \\
\hline $\mathrm{FEV}_{1} / \mathrm{FVC}, \%$ & $79.80 \pm 6.06$ & $55.25 \pm 12.34$ & $42.67 \pm 15 \cdot 51$ & $<0.001$ \\
\hline DLCO, $\%$ predicted & $100.40 \pm 36.92$ & $81.90 \pm 23.29$ & $80.05 \pm 25.76$ & 0.288 \\
\hline
\end{tabular}

Values are presented as mean $\pm \mathrm{SD}$ or number $(\%)$.

COPD, chronic obstructive pulmonary disease; BMI , body mass index; FVC, forced vital capacity; FEV $_{1}$, forced expiratory volume in 1 second; $\mathrm{FEV}_{1} / \mathrm{FVC}$, forced expiratory ratio; DLCO, diffusion capacity of the lung for carbon monoxide.

Table 2. Distribution of Nrf2, IL-6, SP-D, and CRP levels in each group

\begin{tabular}{lccccccc}
\hline & Control & COPD-stable & $\begin{array}{c}\text { COPD- } \\
\text { exacerbation }\end{array}$ & $\begin{array}{c}\text { Shapiro } \\
\text {-Wilk }\end{array}$ & Levene's test & $\begin{array}{c}\text { Jonckheere- } \\
\text { Terpstra } p \text { value }\end{array}$ \\
\hline $\mathrm{Nrf2}, \mathrm{ng} / \mathrm{mL}$ & $12.71 \pm 2.08$ & $14.08 \pm 1.87$ & $15.22 \pm 3.05$ & 0.06 & 0.06 & $0.006^{\mathrm{a}}$ & 0.002 \\
$\mathrm{IL}-6, \mathrm{pg} / \mathrm{mL}$ & $10.62 \pm 5.20$ & $12.34 \pm 6.85$ & $25.58 \pm 32.19$ & $<0.001$ & $<0.001$ & $0.653^{\mathrm{b}}$ & - \\
$\mathrm{SP}-\mathrm{D}, \mathrm{pg} / \mathrm{mL}$ & $85.13 \pm 41.45$ & $102.32 \pm 70.01$ & $162.03 \pm 128.73$ & $<0.001$ & $<0.001$ & $0.124^{\mathrm{b}}$ & - \\
$\mathrm{CRP}, \mathrm{mg} / \mathrm{dL}$ & $1.55 \pm 3.26$ & $2.87 \pm 4.14$ & $5.15 \pm 6.17$ & $<0.001$ & 0.080 & $0.001^{\mathrm{b}}<0.001$ \\
\hline
\end{tabular}

Values are presented as mean $\pm \mathrm{SD}$.

$\mathrm{Nrf2}$, nuclear factor erythroid 2-related factor 2; IL-6, interleukin 6; SP-D, surfactant protein D; CRP, C-reactive protein; COPD, chronic obstructive pulmonary disease.

${ }^{a}$ Analysis of variance.

${ }^{\mathrm{b}}$ Kruskal-Wallis test.

Nrf2 levels and the correlations between Nrf2 and other important clinical parameters in patients with COPD. Oxidative stress from smoking and air pollutants plays a major role in the development and progression of
COPD [11]. Moreover, oxidative stress is markedly different according to the severity of COPD, and is particularly increased during exacerbation [12]. In addition, airway inflammation caused by excessive oxidative stress 

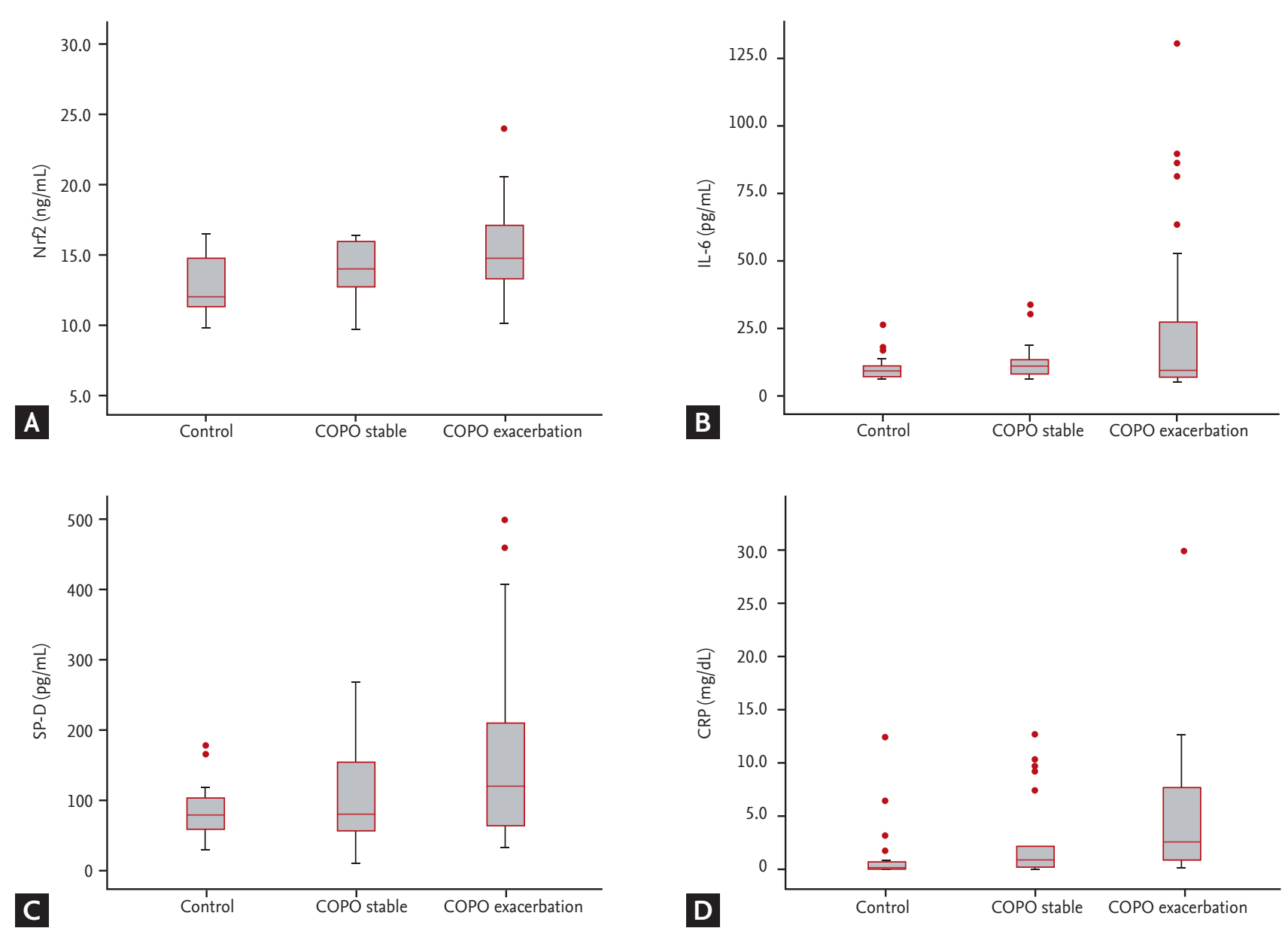

Figure 1. Distribution of the plasma levels of (A) nuclear factor erythroid 2-related factor 2 (Nrf2), (B) interleukin 6 (IL-6), (C) surfactant protein D (SP-D), and (D) C-reactive protein (CRP) in the control group, and the chronic obstructive pulmonary disease (COPD)-stable and COPD-exacerbation groups.

during exacerbation results in deteriorated pulmonary function in patients with COPD [13]. In our study, we observed a gradual decline of respiratory functions and frequent exacerbations according to the disease severity of COPD.

There are several antioxidant systems against oxidative stress in the human body and airways. Nrf2 is one of the most important antioxidant molecules to maintain proteostasis and lung tissue survival [14]. However, whether Nrf2 has a protective role in patients with COPD has been controversial. Several studies have focused on $\mathrm{Nrf} 2$ downregulation and the activity of the Nrf2-directed antioxidant system in COPD patients $[15,16]$. Some authors have suggested that inadequate Nrf2/ARE signaling may amplify destructive inflammatory responses, resulting in disease progression. However, a recent study on peripheral blood mononuclear cells (PBMCs) of patients with ex-smoker COPD reported increased expression (at both the mRNA and protein levels) of both Nrf2 and the related gene heme oxygenase-1 (HO-1) in COPD compared to non-COPD subjects [17]. Persistent oxidative stress in airways after smoking cessation induces sequential Nrf2-directed antioxidant responses. Activated Nrf2 stimulates the expression of antioxidant and detoxification genes that protect the lung parenchyma from chronic oxidative stress. Similarly, we showed the tendency of plasma Nrf2 levels to increase according to the disease state of COPD compared to the control group of the current study. In particular, there was a significant difference between the control group and the 

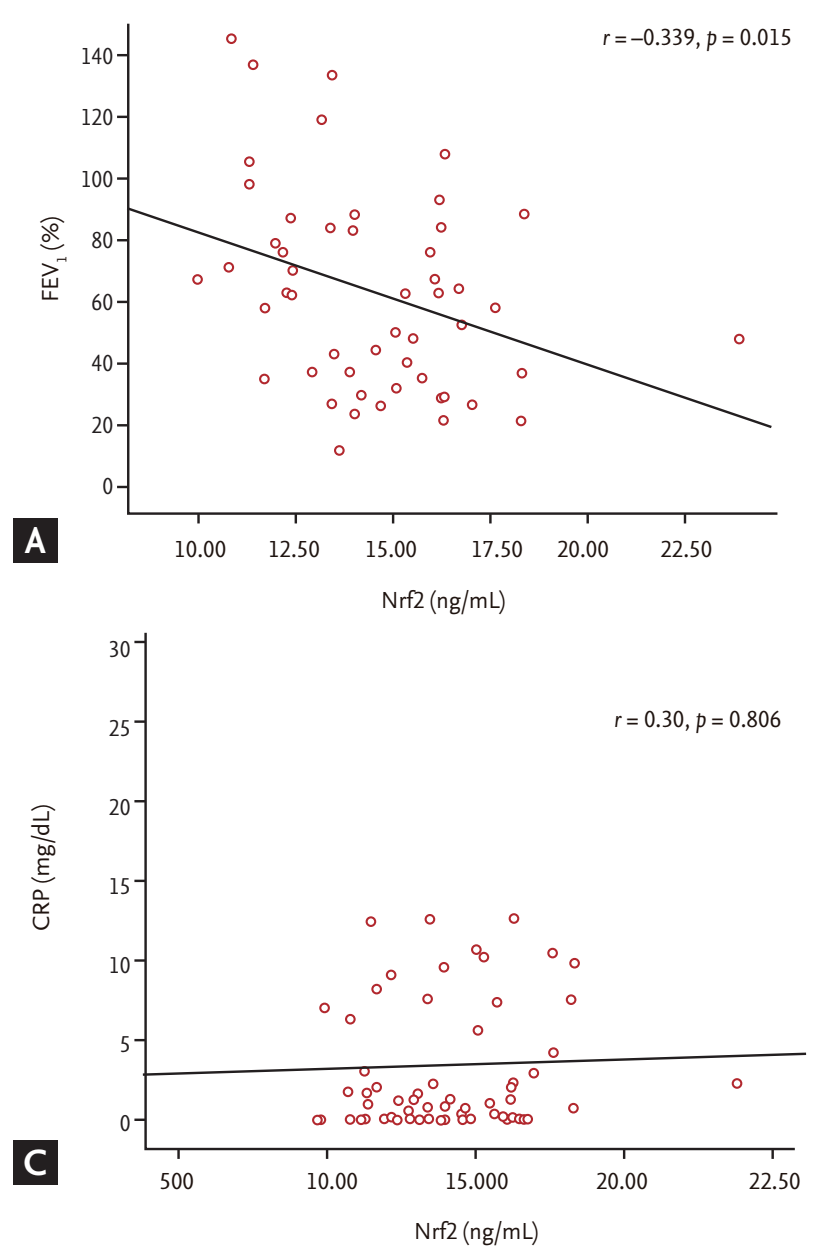

COPD-exacerbation group. Our results suggested that the antioxidant mechanism through Nrf2 activation in COPD-exacerbation was dramatically up-regulated, compared with that in the normal condition. We assume that, in the exacerbation state, there is either excessive and chronic oxidative stress or exorbitant antioxidant responses beyond the regular redox mechanisms, which result in the severe deterioration of respiratory function.

Current concepts regarding COPD are that it is not a limited airway disease but a whole systemic inflammatory disease. A number of studies have been conducted to determine the probable systemic inflammatory markers for the diagnosis and prediction of the disease process in patients with COPD. In the present study, we assessed several systemic inflammatory markers including IL-6, SP-D, and CRP in different severities of COPD. IL-6 is a proinflammatory cytokine and one of the key immune-modulating molecules in systemic

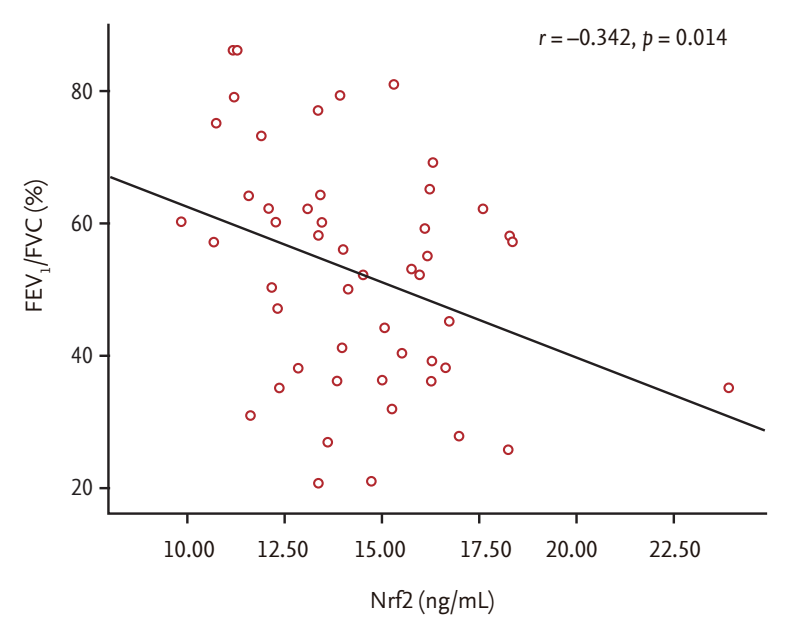

Figure 2. Correlations between nuclear factor erythroid 2-related factor 2 (Nrf2) levels in, and other clinical parameters (A) forced expiratory volume in 1 second $\left(\mathrm{FEV}_{1}\right),(\mathrm{B})$ $\mathrm{FEV}_{1}$ /forced vital capacity (FVC), and (C) C-reactive protein (CRP) level of, the study population.

inflammatory responses in COPD [18]. Liang et al. [19] demonstrated that the blood IL-6 level was significantly higher in patients with COPD-exacerbation than in stable COPD and control patients. In addition, Foschino Barbaro et al. [20] showed similar results in the airway epithelial cells of COPD patients. SP-D is a member of the collectin family and participates in the innate immune regulatory response of the lungs [21]. This protein is produced predominantly in the lungs; however, during inflammation, it translocates into the blood and can be found in the serum [22]. Therefore, it is considered to be a strong candidate for a biomarker of several pulmonary diseases. Ju et al. [23] suggested that SP-D may be a potential systemic biomarker for COPD since it showed different serum SP-D levels according to the disease state of the patient. CRP, another major systemic inflammatory marker, has been studied in detail in COPD. CRP is an acute-phase protein and reflects the 
total systemic inflammation burden in patients with COPD [24]. Broekhuizen et al. [25] revealed correlations between raised CRP levels and functional impairment in COPD. In our study, we showed the tendencies of these systemic inflammatory markers, including Nrf2, to increase according to disease severity. However, significant differences were detected only in the Nrf2 and CRP levels between the control group and the COPD groups. Because of complexities of the immune mechanism and diverse connectivity among inflammatory molecules in COPD progression, it has been difficult to confirm certain circulating molecules as indicators of COPD disease severity. Therefore, we needed to add other significant studies in order to confirm the possibility of using plasma Nrf2 and CRP as useful biomarkers of COPD.

We evaluated the correlations between the Nrf2 and CRP levels and meaningful pulmonary function parameters that were significantly different according to the severity of COPD. Eventually, we observed strong negative correlations between the plasma level of Nrf2 and $\mathrm{FEV}_{1}(\%)$ and $\mathrm{FEV}_{1} / \mathrm{FVC}(\%)$. However, the serum CRP level did not show any correlations with these parameters. These results suggest that the plasma level of Nrf2 could be a potential biomarker of COPD.

There were several limitations to this study. First, we were unable to enroll a large number of patients. This was partly because we excluded patients who had distinct pneumonia, which might markedly affect the activation of systemic inflammation. However, by so doing, we were able to control the unexpected changeability of inflammatory markers due to pneumonia. Second, we did not describe the reference levels of each biomarker. To overcome this, we assessed their levels according to different stages (control vs. COPD-stable vs. COPD-exacerbation) and demonstrated the stepwise increase of the parameters according to the inflammatory state. Finally, there are not enough studies in the literature to validate the plasma Nrf2 levels. Under basal conditions, Nrf2 is destroyed by proteasomal degradation, through association with its cytosolic inhibitor, Kelch-like ECH-associated protein 1 (KEAP1). In response to oxidative stress, $\mathrm{Nrf} 2$ dissociates from $\mathrm{KEAP}_{1}$, translocates into the nucleus, and activates several antioxidant genes [5]. Detection of the activity of Nrf2 using cell lysates via tissues or PBMCs is considered to be a key technique. However, it is relatively cost-ineffective and even more invasive.
Therefore, its clinical application to measure Nrf2 activity has been limited, and studies where plasma Nrf2 has been utilized to estimate its activity have been rare, to date. In the present study, we evaluated the plasma Nrf2 levels in patients with COPD and showed a gradual increase according to disease severity, in the same manner as other conventional methods. To the best of our knowledge, this is the first study to assess Nrf2 activity in the plasma of COPD patients. We consider that the leakage of Nrf2 from the nucleus during the process of cell necrosis or apoptosis during inflammation would be detected in the blood samples of patients. Therefore, it may be more useful and easier to assess the level of plasma Nrf2 in order to evaluate the degree of indirect systemic inflammation in COPD. A further large-scale study will be needed to validate these results and to determine the precise mechanisms of Nrf2 in COPD.

In conclusion, this study showed that plasma Nrf2 levels increased gradually according to disease severity and systemic inflammation in COPD, and that Nrf2 had significant correlations with other important clinical parameters of COPD, such as $\mathrm{FEV}_{1}(\%)$ and $\mathrm{FEV}_{1} /$ FVC (\%). Therefore, further clinical studies are needed to clarify the precise role and mechanism of action of plasma Nrf2 by systemic inflammatory status in patients with COPD.

\section{KEY MESSAGE}

1. Current concepts regarding chronic obstructive pulmonary disease (COPD) are that it is not a limited airway disease but a whole systemic inflammatory disease.

2. Plasma nuclear factor erythroid 2-related factor 2 (Nrf2) levels gradually increased in line with disease severity and the extent of systemic inflammation in patients with COPD.

3. Assessing the level of plasma Nrf2 can be a useful method to evaluate the degree of indirect systemic inflammation in COPD according to disease severity.

\section{Conflict of interest}

No potential conflict of interest relevant to this article was reported. 


\section{REFERENCES}

1. Mathers CD, Loncar D. Projections of global mortality and burden of disease from 2002 to 2030. PLoS Med 2006;3:e442.

2. Guarascio AJ, Ray SM, Finch CK, Self TH. The clinical and economic burden of chronic obstructive pulmonary disease in the USA. Clinicoecon Outcomes Res 2013;5:235245 .

3. Mannino DM, Higuchi K, Yu TC, et al. Economic burden of COPD in the presence of comorbidities. Chest 2015;148:138-150.

4. Kensler TW, Wakabayashi N, Biswal S. Cell survival responses to environmental stresses via the Keap1-Nrf2ARE pathway. Annu Rev Pharmacol Toxicol 2007;47:89-116.

5. Boutten A, Goven D, Artaud-Macari E, Boczkowski J, Bonay M. NRF2 targeting: a promising therapeutic strategy in chronic obstructive pulmonary disease. Trends Mol Med 2011;17:363-371.

6. Rodriguez-Roisin R. Toward a consensus definition for COPD exacerbations. Chest 2000;117(5 Suppl 2):398S-401S.

7. Celli BR, Barnes PJ. Exacerbations of chronic obstructive pulmonary disease. Eur Respir J 2007;29:1224-1238.

8. Vestbo J, Hurd SS, Agusti AG, et al. Global strategy for the diagnosis, management, and prevention of chronic obstructive pulmonary disease: GOLD executive summary. Am J Respir Crit Care Med 2013;187:347-365.

9. Burge PS, Calverley PM, Jones PW, Spencer S, Anderson JA, Maslen TK. Randomised, double blind, placebo controlled study of fluticasone propionate in patients with moderate to severe chronic obstructive pulmonary disease: the ISOLDE trial. BMJ 2000;320:1297-1303.

10. Laszlo G. Standardisation of lung function testing: helpful guidance from the ATS/ERS Task Force. Thorax 2006;61:744-746.

11. Antus B, Kardos Z. Oxidative stress in COPD: molecular background and clinical monitoring. Curr Med Chem 2015;22:627-650.

12. Stanojkovic I, Kotur-Stevuljevic J, Milenkovic B, et al. Pulmonary function, oxidative stress and inflammatory markers in severe COPD exacerbation. Respir Med 2011;105 Suppl 1:S31-S37.

13. Seemungal TA, Donaldson GC, Bhowmik A, Jeffries DJ, Wedzicha JA. Time course and recovery of exacerbations in patients with chronic obstructive pulmonary disease. Am J Respir Crit Care Med 2000;161:1608-1613.
14. Cantin AM, Richter MV. Cigarette smoke-induced proteostasis imbalance in obstructive lung diseases. Curr Mol Med 2012;12:836-849.

15. Goven D, Boutten A, Lecon-Malas V, et al. Altered Nrf2/ Keapı-Bachı equilibrium in pulmonary emphysema. Thorax 2008;63:916-924.

16. Suzuki M, Betsuyaku T, Ito Y, et al. Down-regulated NF-E2-related factor 2 in pulmonary macrophages of aged smokers and patients with chronic obstructive pulmonary disease. Am J Respir Cell Mol Biol 2008;39:673682.

17. Fratta Pasini AM, Ferrari M, Stranieri C, et al. Nrf2 expression is increased in peripheral blood mononuclear cells derived from mild-moderate ex-smoker COPD patients with persistent oxidative stress. Int J Chron Obstruct Pulmon Dis 2016;11:1733-1743.

18. Yanbaeva DG, Dentener MA, Creutzberg EC, Wouters EF. Systemic inflammation in COPD: is genetic susceptibility a key factor? COPD 2006;3:51-61.

19. Liang R, Zhang W, Song YM. Levels of leptin and IL-6 in lungs and blood are associated with the severity of chronic obstructive pulmonary disease in patients and rat models. Mol Med Rep 2013;7:1470-1476.

20. Foschino Barbaro MP, Carpagnano GE, Spanevello A, Cagnazzo MG, Barnes PJ. Inflammation, oxidative stress and systemic effects in mild chronic obstructive pulmonary disease. Int J Immunopathol Pharmacol 2007;20:753763.

21. Sorensen GL, Hjelmborg Jv, Kyvik KO, et al. Genetic and environmental influences of surfactant protein D serum levels. Am J Physiol Lung Cell Mol Physiol 2006;290:L1010-L1017.

22. Bowler RP. Surfactant protein D as a biomarker for chronic obstructive pulmonary disease. COPD 2012;9:651653.

23. Ju CR, Liu W, Chen RC. Serum surfactant protein D: biomarker of chronic obstructive pulmonary disease. Dis Markers 2012;32:281-287.

24. Sin DD, Man SF. Why are patients with chronic obstructive pulmonary disease at increased risk of cardiovascular diseases? The potential role of systemic inflammation in chronic obstructive pulmonary disease. Circulation 2003;107:1514-1519.

25. Broekhuizen R, Wouters EF, Creutzberg EC, Schols AM. Raised CRP levels mark metabolic and functional impairment in advanced COPD. Thorax 2006;61:17-22. 